\title{
Thymoma 20 Years After Hodgkin's Lymphoma Radiation Therapy: A Case Study
}

\author{
Shauna Wentzella, b, Mary Ryan ${ }^{\mathrm{a}}$
}

\begin{abstract}
We present a case of a 48-year-old female who, over the course of 2 years, developed a recurrence of thymoma. She presented in 2013 with nausea and scalp pruritis, which progressed to generalized body pruritis. Examination was normal except for erythroderma noted on both arms and trunk sparing the lower limbs. A CT scan was performed which showed a $2.5 \mathrm{~cm}$ anterior mediastinal lymph node mass. A biopsy was performed which indicated a stage $2 \mathrm{~b}$ thymoma. Her previous medical history was significant for a $1 \mathrm{~A}$ nodular lymphocyte predominant Hodgkin's lymphoma in 1992 for which she received mantle field radiation. Her presenting symptoms were thought to be of paraneoplastic origin. Thymectomy and excision of the pleural seeding occurred in 2013 with good results. A year later, a followup PET scan revealed a recurrence of her thymoma. Chemotherapy was started with good regression; however, in 2015 the thymoma had spread to the lungs, adjacent pleura and pericardium. Second line chemotherapy was initiated. This case report highlights the possible association between mantle field radiation for Hodgkin's lymphoma leading to a future thymoma. It emphasizes the need to consider secondary malignancies in the differential diagnosis for patients presenting with unexplained symptoms and a previous history of radiation therapy.
\end{abstract}

Keywords: Mantle field radiation; Malignancy; Hodgkin's lymphoma; Secondary malignancy

\section{Introduction}

This case was written to highlight the probable relationship between the development of a thymoma following mantle field radiation therapy for Hodgkin's lymphoma (HL). It is also meant to educate readers about the importance of secondary malignancies occurring following radiation therapy for HL.

Manuscript accepted for publication March 07, 2016

aDepartment of Endocrinology, Barrington's Hospital, Limerick, Ireland ${ }^{\mathrm{b}}$ Corresponding Author: Shauna Wentzell, Department of Endocrinology, Barrington’s Hospital, Limerick, Ireland. Email: Wentzell.shauna@gmail.com

doi: http://dx.doi.org/10.14740/jmc2466w

\section{Case Report}

A 48-year-old woman presented to the Medical Oncology Clinic with nausea, generalized pruritis and rash covering her arms and torso. On further questioning, she mentioned the rash started locally on the scalp and subsequently spread over her body. She does not consume alcohol and is a smoker.

Her physical examination was essentially normal except for the erythroderma noted on her arms and trunk. She had a clear chest, no lymphadenopathy and a soft, non-tender abdomen with no organomegaly.

Her medical history was significant for type 1 A lymphocyte predominant HL in 1992 for which she received whole chest mantle field radiation as a primary treatment.

\section{Investigations}

Ultrasound revealed a normal abdomen and pelvis; however, a CXR and subsequent chest CT scan identified a $2.5 \mathrm{~cm}$ mass in the anterior mediastinum lateral to the aortic arch. She was referred to a cardiothoracic surgeon who biopsied the mass. This revealed a stage $2 \mathrm{~B}$ thymoma.

\section{Treatment}

The thymoma was resected in May 2013 with excision of pleural seedings. A PET scan in July 2014 showed a large left pleural effusion with masses in the left lung base measuring up to 5 $\mathrm{cm}$. There were also sclerotic lesions at the level of T5 and the left iliac bone representing small bone islands. A repeat biopsy was obtained demonstrating a recurrence with high proliferative index. A more aggressive clinical course was undertaken and she was started on cisplatin, doxorubicin and cyclophosphamide chemotherapy. A repeat PET scan was scheduled as a 12week follow-up to monitor disease status following treatment.

\section{Outcome and follow-up}

She initially had good response to her chemotherapy treatment and according to a multidisciplinary team meeting in November 2014 she was deemed disease free. A follow-up PET scan performed on October 2015 showed progression of the disease 
to her left hemithorax, pleura and pericardium. Second line therapy was suggested to include taxane based chemotherapy as the affected field was deemed too large for either surgery or radiation.

\section{Discussion}

Radiation therapy is a procedure commonly used to treat malignancies. It has been very successful following its implementation; however, the potential long term consequences of secondary malignancies as a result of radiation must be acknowledged. With better patient care and cancer therapies available, patients are living longer and the dangers of secondary malignancies cannot be ignored.

Mantle field radiation is very useful when treating localized HL. Most often it is given in conjunction with chemotherapy; however, if the disease itself is of nodular lymphocyte predominance (as in the case of our patient), radiation itself is sufficient as these tumors tend to grow at a very slow pace and is less responsive to chemotherapy.

With survival rates of HL increasing from $75 \%$ in 1971 to $91 \%$ in 2011 [1], secondary malignancies from previous mantle field radiation are becoming a growing concern. There is good support that radiation related secondary cancer risk among HL survivors is largely based on previous radiation therapy [2].

It should also be noted that risk of a secondary malignancy after HL continues to rise 15 - 20 years following therapy and does not appear to plateau. After 15 and 20 years, there is a $2.3 \%$ and $4.0 \%$ risk of secondary malignancy per person per year. This risk is even further increased when treatment was combined with chemotherapy [3].

Due to the large radiation field and high doses previously given when treating patients with mantle radiation, newer techniques have since been developed. These newer modalities are created with the intention of decreasing patient radiation dose and surrounding tissue exposure. Involved field radiotherapy (IFRT) is one such newer modality and has shown some evidence for reducing the late effects of treatment [1]. With newer radiation therapies coming to the forefront as possible alternative radiation treatment for HL, there is strong evidence that this will reduce the secondary malignancy burden. A study conducted by Eng-Siew et al [4] states that with the IFRT alone, the estimated relative risk for radiation induced breast cancer and lung cancer decreased by $64 \%$ and $36-67 \%$, respectively. With these findings, it is hypothesized that this will also decrease the future occurrence of other solid organ secondary malignancies, such as thymoma.

Ultimately, the concern for secondary malignancy will never disappear. It must always be considered as a long term risk when determining treatment for HL patients. With the use of mantle field radiation slowly being replaced with safer alternatives, there is evidence suggesting that the overall secondary malignancy burden will be decreased. That being said, these newer modalities have not yet fully replaced old therapies. Therefore, the importance of including secondary malignancies in a differential diagnosis for a patient with a past medical history of HL treated with radiation must be considered.

\section{Learning points}

There is a probable relationship between mantle field radiation for $\mathrm{HL}$ and the future development of a thymoma.

Secondary malignancy should be considered in the differential for a patient presenting with unexplained symptoms following previous mantle field radiation therapy.

There is support that newer radiation therapy modalities for HL reduce the secondary malignancy burden.

\section{Funding}

None.

\section{Disclosure}

None.

\section{Competing Interests}

None.

\section{References}

1. Hodgkin lymphoma survival statistics. Cancer Research UK. Available at: http://www.cancerresearchuk.org/ health-professional/cancer-statistics/statistics-by-cancertype/hodgkin-lymphoma/survival.

2. Hodgson DC, Koh ES, Tran TH, Heydarian M, Tsang R, Pintilie $\mathrm{M}, \mathrm{Xu} \mathrm{T}$, et al. Individualized estimates of second cancer risks after contemporary radiation therapy for Hodgkin lymphoma. Cancer. 2007;110(11):2576-2586.

3. Ng AK, Bernardo MV, Weller E, Backstrand K, Silver B, Marcus KC, Tarbell NJ, et al. Second malignancy after Hodgkin disease treated with radiation therapy with or without chemotherapy: long-term risks and risk factors. Blood. 2002;100(6):1989-1996.

4. Koh ES, Tran TH, Heydarian M, Sachs RK, Tsang RW, Brenner DJ, Pintilie M, et al. A comparison of mantle versus involved-field radiotherapy for Hodgkin's lymphoma: reduction in normal tissue dose and second cancer risk. Radiat Oncol. 2007;2:13. 